A rare disease is one that affects a small percentage of people in a given population and is largely genetic in origin. Taken together they affect millions of people around the world. The cost of genome sequencing has fallen one-million-fold in the past several years; in principle, this wealth of genome sequence data should accelerate progress in biomedicine, making it possible to integrate genomic and clinical information to reveal the genetic basis of inherited diseases, amongst many other applications. However, we are not organised to seize this extraordinary opportunity, nor are we on a path to do so. Two international consortia, the International Rare Diseases Research Consortium (IRDiRC) and the Global Alliance for Genomics and Health (GA4GH), have recognised rare diseases as a remarkable opportunity for advancement in this realm on a world-wide scale. The collaborative efforts of these two international consortia to advance rare disease gene discovery will be reviewed, including initiatives such as Matchmaker Exchange (www. matchmakerexchange.org).

\section{HEALTH ECONOMICS OF EXOME SEQUENCING FOR RARE DISORDERS}

Deborah Schofield

University of Sydney, NSW, Australia

Rare genetic disorders can have devastating health impacts on patients. A sometimes overlooked area of significant impact is the cost of the disorders. The results of a study of the relative cost of exome sequencing compared to usual care are presented, along with a review of the breadth of the costs of monogenic childhood disorders both within and beyond the health system.

\section{FACIAL DIAGKNOWSIS}

Gareth Baynam $^{1,2,3,4,5,6}$

${ }^{1}$ Genetic Services of Western Australia, Princess Margaret \& King Edward Memorial Hospitals, ${ }^{2}$ Office of Population Health Genomics, Department of Health, Public Health and Clinical Services Division, ${ }^{3}$ School of Paediatrics and Child Health, University of Western Australia, ${ }^{4}$ Institute for Immunology and Infectious Diseases, Murdoch University, ${ }^{5}$ Western Australian Register of Developmental Anomalies, WA Department of Health, and ${ }^{6}$ Telethon Kids Institute, WA, Australia

Our face is a biological billboard that reflects our physical and mental wellness, our ageing and our disease.

Investigating our faces in their 3-dimensional form using facial surface imaging coupled with data processing [3D facial analysis (3DFA)] is at the edge of medical innovation and translation. Image capture takes less than 2 milliseconds; it is non-invasive, non-irradiating, exquisitely sensitive, robust, transportable and inexpensive when compared to other imaging techniques. Increasingly 3DFA is used to unlock knowledge from the facial surface through deep phenotyping for precision medicine applications including: (i) facial diagnostics, particularly for objective diagnosis of genetic and rare diseases which cumulatively affect up to 1 in 12 people; (ii) facial surgical planning and audit; (iii) facial medical device design; and (iv) therapeutic (drug) monitoring, specifically, as an objective solution to rapidly assess the response or failure of drugs in clinical use or in trial.

This presentation will focus on clinical and translational diagnostic applications, with and without partnership with (epi) genomic testing, and international networks. 